\title{
Krise und Alternativen im europäischen Integrationsprozess
}

Was verbindet man in 2014 schon noch mit der Europäischen Union (EU)? Neben dem Wegfall der Grenzen und einem Leben auf einem friedlichen Kontinent dominieren doch vor allem Unsicherheiten und Ängste: Unsicherheiten über das Verbot von Glühbirnen, Ölkännchen und Mentholzigaretten, Ängste vor dem Verlust sozialer Standards, steigender Preise und des „triple A“, steigende Armutsmigration und Jugendarbeitslosigkeit, der Ausstieg aus dem Euro - Krisenstimmung. Aber worin besteht die Europakrise? Oder anders formuliert: Gibt es überhaupt die Europakrise?

\section{Eine europäische Wirtschaftskrise}

Die Präsidentin Litauens und Trägerin des internationalen Karlspreises, Dalia Grybauskaite (2013), brachte es auf den Punkt: Wir haben keine Eurokrise. Der aktuelle Kurs des Euros liegt seit Monaten deutlich über 1,30 US-Dollar. Schauen wir uns den Wert über die letzten Jahre an, so hat sich der Euro als vergleichsweise stabile Währung erwiesen. Was ist es also dann?

Aus ökonomischer Sicht haben wir uns in den letzten sechs Jahren seit der „Lehmann-Pleite" von einer amerikanischen Immobilienkrise hin zu einer europäischen Banken- und Schuldenkrise bewegt. Verschärft wurde das Ganze durch fehlende Haushaltsdisziplin und einen Mangel an Wettbewerbsfähigkeit.

Allerdings haben die Probleme in Irland nichts mit einem Fehlen von Strukturreformen zu tun. Auch Spanien ist im Moment immer noch weniger verschuldet als Deutschland. Schaut man also genauer hin, handelt es sich eigentlich um sehr länderspezifische makroökonomische Probleme: In Griechenland geht es vor allem um einen Staatsbankrott, der abgewendet werden musste; in Italien geht es um überfällige Wirtschaftsreformen; in Spanien und Irland resultiert die Krise aus der Schwäche der Banken und in Portugal aus der negativen Produktivitätsentwicklung der Gesamtwirtschaft über die letzten Jahrzehnte. 


\section{Eine europäische Vertrauenskrise}

Alle diese ökonomischen Probleme sind spürbar. Sie gehen einher mit für die Menschen teilweise sehr schmerzvollen, innenpolitischen Reformen. Parallel dazu schwindet das politische Vertrauen in die EU, die Krise zu lösen. Das europäische Projekt verliert an Überzeugungskraft - mittlerweile in der Bevölkerung fast aller Mitgliedstaaten. Laut Eurobarometer schaut fast die Hälfte aller EU-Bürger pessimistisch in die Zukunft. ${ }^{1}$ Nicht gerade beruhigende Zahlen mit Blick auf die bevorstehende Wahl zum Europäischen Parlament. Auf nationaler Ebene hat die Vertrauenskrise seit Ende 2008 bereits zu insgesamt 19 Regierungswechseln geführt. ${ }^{2}$

Wir haben es aber in der Bevölkerung nicht erst seit der Finanzkrise mit einer handfesten europäischen Vertrauenskrise zu tun - seit langem schon begleitet eine schwelende Integrationsmüdigkeit (eine sogenannte ,fatigue") den europäischen Einigungsprozess. In den 1980er Jahren machte zum Beispiel zwischen 1973 und 1984 das „Gespenst der Eurosklerose“ die Runde (Olsen 1996: 73 ff.).

In letzter Zeit jedoch hat die Vertrauenskrise mit dem verstärkten Auftreten europa- bzw. eurokritischer Parteien eine nеue Qualität erreicht. Die aktuelle Krisenstimmung bereitet den Nährboden für europaskeptische Parteien - rechts und links. Man denke an die letzten Wahlerfolge der „Wahren Finnen“ oder der italienischen „Bewegung Fünf Sterne“. Aber auch in Deutschland wird durch die Gründung der „Alternative für Deutschland“ (AfD) mit dem liberalen Konsensus hinsichtlich der EU gebrochen. Die Voraussetzungen für eine eurokritische Partei rechts der Union sind mit Blick auf die letzte Bundestagswahl jetzt auch in Deutschland gegeben.

\section{Eine europäische Existenzkrise}

Das wiederum rückt David Cameron Europarede (2013) in den Fokus - von besonderem Interesse hierbei seine Motive. Cameron verstand es seine lang erwartete Europa-Rede für seine innenpolitischen Ziele zu instrumentalisieren. Zum einen, indem er seine eigene Partei (wenn auch nur kurzfristig) hinter sich scharen konnte, und zum anderen, indem er sich den Anhängern der europaskeptischen und rechtspopulistischen UKIP annäherte. Allerdings zu einem Preis: dem Zugeständnis, bis 2017 ein britisches Referendum über die Zukunft des Landes in der EU abzuhalten. Ein Referendum, das in einem möglichen EU-Austritt des Vereinigten Kö-

1 http://ec.europa.eu/,http://ec.europa.eu/public_opinion/archives/eb/eb80/eb80_first_de.pdf(Stand: 11.3.2014).

2 Nur in Deutschland, Estland, Lettland, Luxemburg, Österreich, der Niederlande, Polen und Schweden blieben die Regierungen ,stabil“ beziehungsweise fielen aufgrund ausschließlich innenpolitischer Gründe. 
nigreichs münden könnte - das wissen wir spätestens seit den Regionalwahlen im März 2013, bei denen UKIP die regierenden Tories und die oppositionelle LabourPartei in einer Region sogar auf den dritten und vierten Platz verwies. Die letzten Umfragen zeigen, dass im Moment 45 Prozent der Briten für einen EU-Austritt stimmen würden. ${ }^{3}$

Doch je stärker das EU-skeptische Lager in Großbritannien, desto wahrscheinlicher wird eine Abspaltung des europafreundlichen Schottlands. Jene in den letzten Jahren vehement vorgetragenen Unabhängigkeitsbestrebungen, aber auch die Kataloniens, werden die EU schon im September 2014 vor große existentielle Herausforderungen stellen.

\section{Eine europäische Wertekrise}

Freiheit, soziale Gerechtigkeit, demokratische Teilhabe und Rechtsstaatlichkeit hierfür steht die Europäische Union und ihre Mitgliedstaaten (Artikel 2 und 3 des EU-Vertrags). Seit einigen Jahren allerdings mehren sich Verstöße gegen den europäischen Wertekanon. Mitgliedstaaten der EU verstoßen gegen die Presse- und Medienfreiheit (Italien und Ungarn); zu erwähnen sind auch die Maßnahmen der Abschiebung von Roma in Frankreich, die versuchte Entmachtung des Verfassungsgerichts in Rumänien, der Austausch höherer Beamter in der Justiz in Ungarn, oder aber die „Operation Tempora“ des britischen Geheimdienstes zur Überwachung des weltweiten Telekommunikations- und Internet-Datenverkehrs (Stichwort Datenspeicherung). ${ }^{4}$

Nachdem ein Land Mitglied der EU geworden ist (und damit zum Beitrittsdatum die sogenannten Kopenhagen-Kriterien erfüllt hat), bestehen so gut wie keine Möglichkeiten auf europäischer Ebene, beispielsweise Defizite im Justizwesen oder Verfassungsänderungen in einem frühen Stadium zu thematisieren und Reformen zu verlangen. EU-Kommissarin Viviane Reding nennt dies seit einiger Zeit das „Kopenhagen-Dilemma“ (2013). Europa befindet sich seit Jahren in einer substanziellen Wertekrise.

3 http://ukpollingreport.co.uk/blog/archives/category/europe (Stand: 11.3.2014).

4 Artikel 16 des Vertrags über die Arbeitsweise der EU legt fest: ,Jede Person hat das Recht auf Schutz der sie betreffenden personenbezogenen Daten.“ 


\section{Eine europäische Compliancekrise}

Viele Mitgliedstaaten halten sich zudem zu oft nicht an europäische Vereinbarungen. Bestehendes EU-Recht wird von den EU-Mitgliedstaaten systematisch verspätet und inhaltlich falsch umgesetzt. Hierbei handelt es sich um ein europaweites Problem. In 2012 waren es mehr als 2.000 Fälle, in denen gegen EU-Mitgliedstaaten sogenannte Vertragsverletzungsverfahren angestrengt wurden. 45 Prozent aller Vertragsverletzungsverfahren betrafen die Bereiche Umwelt und Steuern. ${ }^{5}$

Die Mitgliedstaaten riskieren hierbei neben Bußgeldern in Millionenhöhe vor allem auch einen suboptimal funktionierenden Binnenmarkt. Die Nichtumsetzung von EU-Recht schafft Unsicherheiten bei Unternehmen und Bürgern, unterminiert die Legitimität von EU-Rechtsvorschriften und des Brüssler Entscheidungsfindungsapparates (Kaeding 2012). Das wiederum ist problematisch. Denn um Europa aus der wirtschaftlichen Stagnation zu führen, kommt gerade dem europäischen Binnenmarkt eine Schlüsselrolle zu. Ein funktionierender Binnenmarkt garantiert nicht nur den freien Waren-, Personen-, Dienstleistungs- und Kapitalverkehr, sondern stärkt auch die globale Wettbewerbsfähigkeit eines sozialen Europas, nationales Wachstum und Arbeitsplätze (Monti 2010).

\section{Eine europäische institutionelle Krise}

Verstärkt wird diese europäische Wirtschafts-, Vertrauens-, Werte-, Existenz- und Compliancekrise durch ein europäisches Institutionengefüge, das diesen aktuellen Herausforderungen nicht gerecht wird (Scharpf 1999). In Zeiten der Globalisierung - der „,beschleunigten Entnationalisierung“ - bedarf es einer effizienten, flexiblen und demokratisch legitimierten europäischen Governancestruktur. Allerdings dauern die Entscheidungsfindungsprozesse auf europäischer Ebene oft zu lang. 16 Prozent aller Verhandlungen zwischen dem Ministerrat und dem Europäischen Parlament, den beiden EU Gesetzgebern, dauerten in den letzten Jahren im Durchschnitt 28 Monate oder länger (Europäisches Parlament 2012). ${ }^{6} \mathrm{Zu}$ oft ist zudem die Qualität der Entscheidungen gering (Risse/Kleine 2007).

Damit riskiert die EU nicht nur, den Anschluss an die Vereinigten Staaten und die BRIC-Staaten zu verlieren (Stichwort Shanghai-Perspektive), sondern auch, auf

5 http://europa.eu/rapid/press-release_IP-13-127_de.htm (Stand: 15.2.2014).

6 Während der 7. Legislaturperiode (14. Juli $20 \overline{0} 9$ bis 11. März 2014) dauerte die Erste Lesung, in der 84 Prozent aller Gesetzestexte im Mitentscheidungsverfahren angenommen wurden, im Durchschnitt 18 Monate, in der vorzeitigen Zweiten Lesung (8 Prozent aller Gesetzestexte) 28 Monate, der klassischen Zweiten Lesung (6 Prozent) 35 Monate und in der Dritten Lesung (2 Prozent) 30 Monate; vgl. http://www.europarl.europa.eu/code/about/statistics_en.htm (Stand: 24.3.2014). 
bedeutende industrielle Entwicklungen und Neuerungen nicht mehr zeitgerecht reagieren zu können. Folglich muss die Verhandlungs- und Entscheidungsfähigkeit beschleunigt werden - ein Aspekt, der auch mit Blick auf die Verhandlungen über ein Freihandelsabkommen mit Japan und den USA weiter an Bedeutung gewinnen wird. Die Verhandlungen zum Freihandelsabkommen zwischen China und der Schweiz haben etwa knapp zwei Jahre in Anspruch genommen. Im Vergleich dazu dauerten die Verhandlungen zum Freihandelsabkommen zwischen der EU und Südkorea fast doppelt so lang (dreieinhalb Jahre).

Durch die wachsende Zahl der Mitgliedstaaten ist zudem eine europäische „onesize-fits-all“-Lösungsfindung oft nicht flexibel genug. Man kann mit einer „Einheitslösung" den Ansprüchen 28 unterschiedlicher nationaler Systeme nicht immer zwangsläufig gerecht werden. Der Europarechtler Giandomenico Majone brachte es kürzlich auf den Punkt: ,[T]he traditional one-size-fits-all approach to European integration is obsolete", ${ }^{7}$ sozusagen veraltet, technisch überholt.

Aber auch Krisen wie in Libyen, Mali, Syrien, Ägypten oder der Ukraine führen uns vor Augen, dass den EU-Mitgliedstaaten auf internationalem Parkett zu oft der politische Wille fehlt, mit „einer Stimme“ zu sprechen. Es fällt den EU-Staaten schwer, auf nationale Sonderwege zu verzichten.

\section{Eine europäische Legitimationskrise}

Über all dies legt sich ein Mantel des Legitimationsdefizits - das sich sowohl auf europäischer, wie auch auf nationaler Ebene manifestiert (Moravcsik 2008; Bellamy 2010): „The idea behind the notion of a 'democratic deficit' is that decisions in the EU are in some ways insufficiently representative of, or accountable to, the nations and people of Europe“ (Lord 2001: 165).

Im Zuge der Finanzkrise und der Debatte um die verschärfte EU-Haushaltskontrolle kann man sich sicherlich zu Recht fragen: „Wer oder was ist eigentlich Oli Rehn?“ Trotz gewaltiger Eingriffsrechte fehlt es vielen Brüsseler Entscheidungsträgern an der notwendigen Legitimation durch die europäischen Bürger (Neyer 2013). Die Finanzkrise zeigt zudem, dass selbst das Europäische Parlament nicht immer in der Lage war, dieses europäische Demokratiedefizit zu substituieren trotz der Einrichtung eines Sonderausschusses zur Finanzkrise im Oktober 2009. Das, was europäisch gelöst wird, muss aber im europäischen Mehrebenensystem legitimiert werden - durch demokratisch legitimierte europäische und nationale Institutionen.

7 http://www.verfassungsblog.de/de/europe-2023-giandomenico-majone/\#.UyLSsIXjAoM (Stand: 14.3.2014). 
Vielleicht müsste man sich allerdings in dem Zusammenhang erst einmal darüber klar werden, welche Probleme wir auf nationaler Ebene lösen können, und für welche Probleme nationale Lösungsansätze zu kurz greifen: oder anders herum gefragt, welche Probleme müssen bzw. können wir adäquat nur europäisch lösen: Klimawandel, internationaler Terrorismus und Kriminalität, Pandemien, Migration, knapper werdende Rohstoffe, Bevölkerungsrückgang, Proliferation von Massenvernichtungswaffen (Schäuble 2012)? Einige Kompetenzen könnten zudem aus Subsidiaritätsgründen wieder an die Nationalstaaten zurückgegeben werden. Nicht nur David Cameron fordert dies, auch in den Niederlanden hat die Regierung bereits eine „Verschlankungsliste“ angekündigt. Etliche Ministerien sind damit befasst, das europäische Recht zu durchkämmen (Stichwort „repatriation of competences“).

Aber auch auf nationaler Ebene wurde, vor allem durch die Finanzkrise, der politische Wettstreit zwischen Regierung und Opposition praktisch ausgehebelt. Diese „Aushöhlung der nationalen Opposition“ ist in fast allen Mitgliedstaaten festzustellen (Heidbreder 2013). Es handelt sich letzten Endes also um ein ,doppeltes Demokratiedefizit": nicht nur in Brüssel, sondern vor allem auch in den 28 Hauptstädten.

Alles in allem handelt es sich bei der europäischen Krise um sehr unterschiedliche Problemfelder, die sich gegenseitig verstärken und teilweise, wie im Falle der „Aushöhlung der nationalen Opposition", bedingen. Wir haben es seit Jahren in zunehmendem Maße mit einem facettenreichen Problem zu tun, dem Bürger, Politiker und Wissenschaftler (teilweise ohnmächtig) gegenüberstehen. Äußerungen vom „faulen Süden“ und ,italienischen Clowns“, Vorschläge an die Griechen ihre Inseln zu verkaufen, aber auch brennende deutsche Fahnen und Nazi-Vergleiche, zeugen nicht nur von schlechtem Geschmack, sondern von dieser Ohnmacht - und davon, dass die „Dämonen des Nationalismus und Populismus“ (Renan 1882), die die letzten Weltkriege mit ermöglicht haben, nicht verdrängt sind. 100 Jahre nach dem Ausbruch des Ersten Weltkriegs ein denkwürdiger Aspekt.

\section{Wie alternativlos ist der europäische Integrationsprozess?}

Gibt es zur aktuellen Europäischen Union keine Alternative als Krieg? Die Europäische Integrationsforschung hebt vor allem zwei Paradigmen hervor, aus denen sich über die Jahre verschiedene Erklärungen entwickelt haben (Holzinger/Knill/ Peters/Rittberger/Schimmelfennig/Wagner 2005). Sollten wir uns also auf jene Alternativen rückbesinnen? 


\section{Die ,, Vereinigten Staaten von Europa“}

Die „Methode Monnet“ steht für ein supranationales Europa nach dem Muster der „Vereinigten Staaten von Amerika“, um die Vielstaaterei des ,alten“ Kontinents zu beenden. Hierbei bleibt die „finalité politique“ allerdings vieldeutig: „Nicht der institutionelle Gesamtentwurf steht im Vordergrund, sondern einzelne Schritte, die eine Föderation schrittweise aufbauen“"(Wessels 2001: 9).

Besondere Bedeutung bei dieser „Politik der kleinen Schritte“ bekommen konkrete Vorhaben der Wirtschaftspolitik, die ,immer wieder zum strategischen Instrument für einen Prozess hin zu einem politischen System, das politisch unscharf mit einer ,immer engeren Union“ [ever closer Union] beschrieben wird“ (ebd.), genutzt werden und letzten Endes eine Eigendynamik hin zu „mehr Europa“ entfalten.

Neben ökonomischen Instrumenten, die als Hebel dienen, um weitere politische Integrationsschritte umzusetzen, sind es aber vor allem Krisen, die weitere Integrationsschritte erwirken: „,Der Mensch akzeptiert Veränderungen nur unter dem Druck der Notwendigkeit, und die Notwendigkeit nur dann, wenn sie vor einer Krise stehen" (Monnet 1978: 68). Wirtschaftliche Turbulenzen werden demnach immer weitere politische Integrationsschritte notwendig machen. Das von Bundeskanzlerin Merkel im Zusammenhang mit den Griechenland-Hilfspaketen genannte ,alternativlos" steht hierfür exemplarisch.

\section{Vision eines ,Europas der Vaterländer“}

Das alternative Konzept steht für ein intergouvernementales Europa der Nationen. Mitgliedstaaten haben dabei das exklusive Recht das „Europa der Vaterländer“ zu bauen. Damit ist die Grundlage der Einigung Europas die Bewahrung und Stärkung der bestehenden Nationalstaaten mit dem Ziel einer engen Form der zwischenstaatlichen Kooperation. Das europäische Vertragswerk bewahrt im Umkehrschluss die Souveränität der beteiligten Nationen und verzichtet auf supranationale Integrationsschritte. Charles de Gaulle hat dies treffend auf den Punkt gebracht: ,Jedes Volk unterscheidet sich vom anderen durch seine unvergleichliche Persönlichkeit, die unveränderlich und unumkehrbar ist. Wenn Sie wollen, dass die Nationen sich vereinigen, versuchen Sie sie nicht miteinander zu verrühren, wie man Kastanien zu einem Kastanienpüree verrührt [...] Ich glaube also, dass gegenwärtig, nicht mehr als in anderen Epochen, eine Vereinigung Europas keine Fusion der Völker sein kann, sondern dass sie aus ihrer systematischen Annäherung hervorgehen muss“ (1970: 181). 
Die über die letzten Jahre von unterschiedlichen französischen Präsidenten wiederholt vorgetragene Forderung nach einer regierungsähnlichen ,gouvernance économique“ für die Eurozone steht hierfür exemplarisch. Eine „echte Wirtschaftsregierung" soll künftig die Wirtschafts- und Finanzpolitik der Euro-Zone koordinieren, getreu dem Motto: „Wir Staats- und Regierungschefs machen die Politik. Die Kommission setzt das nur um. Punkt."8

\section{Ideologisch geprägt und ,entweder-oder"-Rhetorik}

Mit Blick auf die „Alternativlosigkeit Europas“ bedient sich auch die deutsche öffentliche Debatte häufig dieser beiden Paradigmen der Europäischen Integrationsforschung. Oft wird in dem Zusammenhang die Metapher des „Scheidewegs“ bemüht: Entweder eine ,politischen Union“, ein neuer Konvent und die „Selbstermächtigung der Politik“ (Habermas/Bofinger/Nida-Rümelin 2012) mit dem Ziel einer europäischen Föderation (Fischer 2000) nach der ,postnationalen Revolution“ (Cohn-Bendit/Verhofstadt 2012); oder doch eher ein Staatenbund mit schwächeren europäischen Institutionen, aber einem klaren „Nein an ein deutsches Europa“ (Beck 2012).

Viele dieser Beiträge verfallen immer wieder, wie auch Teile der Europäischen Integrationsforschung, dieser ,entweder-oder“-Rhetorik. Dieses Narrativ wird allerdings der europäischen Realität nicht gerecht. In Demokratien gibt es immer Alternativen. Auch die politische Entscheidung „,mehr Europa“ ist nicht alternativlos. Verhandlungen zwischen 28 Mitgliedstaaten können nur über Kompromisse erreicht werden (auch wenn das vielleicht während nationaler Pressekonferenzen nicht so suggeriert wird). In einer „EU gleichberechtigter Partner“ wird jeder Staat immer Zugeständnisse machen müssen. Hierbei kommt vor allem Deutschland, Frankreich und Polen eine, auch historisch bedingte, besondere Bedeutung zu. Denn entlang der Achse supranational - intergouvernemental liegen sich ihre Positionen traditionell diametral gegenüber. Eine europäische Lösung wird somit immer ein Zwitterwesen mit zwischenstaatlichen und supranationalen Gliedmaßen sein. Auch deshalb heißt es nicht ,entweder-oder": entweder eine Wirtschaftsregierung, geführt von den Nationalregierungen, oder aber eine Stärkung der europäischen Institutionen, wie der Europäische Kommission und der Europäischen Zentralbank mit klarer Abgabe von Macht an Brüssel. Die europäische Integration ist weder schwarz noch weiß, sondern eine Palette vieler Grautöne.

8 So im Jahr 2008 der ehemalige französische Präsident Nicolas Sarkozy am Ende der französischen Ratspräsidentschaft; http://derstandard.at/1369362177973/EU-Wirtschaftsregierung-HollandesMissverstaendnis (Stand: 14.3.2014). 
Krise und Alternativen im europäischen Integrationsprozess

\section{Literatur}

Beck, Ullrich, 2012: Das deutsche Europa: Neue Machtlandschaften im Zeichen der Krise, Berlin

Bellamy, Richard, 2010: Democracy without democracy? Can the EU's democratic 'outputs' be separated from the democratic 'inputs' provided by competitive parties and majority rule?, in: Journal of European Public Policy 17 (1), 2-19.

Cameron, David, 2013: The future of Europe, https:/www.gov.uk/government/wo rld-location-news/the-future-of-europe-prime-ministers-speech-at-bloomberg (Stand: 11.3.2014).

Cohn-Bendit, Daniel/Verhofstadt, Guy, 2012: Für Europa. Ein Manifest, München. Europäisches Parlament, 2012: Tätigkeitsbericht, http://www.europarl.europa.eu/ code/information/activity_reports/activity_report_2009_2011_de.pdf (Stand: 11.3.2014).

Fischer, Joschka, 2000: Vom Staatenverbund zur Föderation - Gedanken über die Finalität der Europäischen Integration, http://whi-berlin.de/documents/ fischer.pdf (Stand: 11.3.2014).

Gaulle, Charles de, 1970: Mémoires d'éspoir. Volume I: Le renouveau (1958-1962), Paris.

Grybauskaite, Dalia, 2013: Rede zur Entgegennahme des Internationalen Karlspreises 2013, http://www.karlspreis.de/preistraeger/2013/rede_von_dalia_gryb auskaite.html (Stand: 11.3.2014).

Habermas, Jürgen/Bofinger, Peter/Nida-Rümelin, Julian, 2012: Für einen Kurswechsel in der Europapolitik, http://www.faz.net/aktuell/habermas-bofinger-ni da-ruemelin-fuer-einen-kurswechsel-in-der-europapolitik-11843182.html (Stand: 3.3.2014).

Heidbreder, Eva, 2013: Brüsseler Illusionen, Süddeutsche Zeitung, Außenansicht, 13.6.2013, 2 (http://www.phil-fak.uni-duesseldorf.de/fileadmin/Redaktion/Inst itute/Sozialwissenschaften/Politikwissenschaft/Dokumente/Heidbreder/SuedD eutsche_130613_Vers2.pdf; Stand: 11.3.2014).

Holzinger, Katharina/Knill, Christoph/Peters, Dirk/Rittberger, Berthold/Schimmelfennig, Frank/Wagner, Wolfgang, 2005: Die Europäische Union: Theorien und Analysekonzepte, München.

Kaeding, Michael, 2012: Towards an Effective European Single Market: Implementing the Various Forms of European Policy Instruments across Member States, Wiesbaden.

Lord, Christopher, 2001: Democracy and Democratisation in the European Union, in: Simon Bromley (Hrsg.), Governing the European Union, London, 165-190. 
Monnet, Jean 1978: Erinnerungen eines Europäers, München.

Monti, Mario, 2010: A new Strategy for the Single Market, http://ec.europa.eu/bepa/ pdf/monti_report_final_10_05_2010_en.pdf (Stand: 11.3.2014).

Moravcsik, Andrew, 2008: The myth of Europe's democratic deficit, in: Intereconomics (November/December), 331-340.

Neyer, Jürgen, 2013: Europa: Integration und Demokratie, http://www.faz.net/akt uell/politik/die-gegenwart/europa-europa-integration-und-demokratie-121405 15.html (Stand: 11.3.2014).

Olson, Mancur, 1996: The varieties of Eurosclerosis: the rise and decline of nations since 1982, in: Nicholas Crafts/Gianni Toniolo (Hrsg.), Economic growth in Europe since 1945, Cambridge.

Reding, Viviane, 2013: Safeguarding the rule of law and solving the "Copenhagen dilemma": Towards a new EU-mechanism, http://europa.eu/rapid/press-release SPEECH-13-348_en.htm (Stand: 11.3.2014).

Renan, Ernest, 1993: Das Plebiszit der Vergeßlichen - über Nationen und den Dämon des Nationalismus, Rede vom 11.3.1882, Frankfurter Allgemeine Zeitung, 27.3.1993.

Risse, Thomas/Kleine, Mareike, 2007: Assessing the Legitimacy of the EU's Treaty Revision Methods, in: Journal of Common Market Studies 45 (1), 69-80.

Scharpf, Wolfgang, 1999: Regieren in Europa - effektiv und demokratisch?, Frankfurt a. M.

Schäuble, Wolfgang, 2012: Rede zur Entgegennahme des Internationalen Karlspreises 2012, http://www.karlspreis.de/preistraeger/2012/rede_von_dr_wolfga ng_schaeuble.html (Stand: 12.3.2014).

Wessels, Wolfgang, 2001: Jean Monnet - Mensch und Methode. Überschätzt und überholt?, in: Reihe Politikwissenschaft des Instituts für Höhere Studien (IHS) Wien, http://www.ihs.ac.at/publications/pol/pw_74.pdf(Stand: 1.3.2014).

Korrespondenzanschrift:

Prof. Dr. Michael Kaeding

Jean Monnet Professor für Europäische Integration und Europapolitik

Institut für Politikwissenschaft

Universität Duisburg-Essen

Lotharstraße 65

47057 Duisburg

E-Mail: michael.kaeding@uni-due.de 